\title{
HDAC6-HSP90途径介导蛋白折叠和降解调控肿瘤细胞 增殖与转移
}

\author{
田点 ${ }^{\dagger}$, 颜晓羽 ${ }^{\dagger}$, 于司杭, 许龙, 赵元馨, 孙连坤 ${ }^{*}$, 苏静* \\ 吉林大学基础医学院病理生理学教研室, 病理生理学教育部重点实验室, 长春 130000 \\ $\dagger$ 同等贡献 \\ *联系人, E-mail: sunlk@jlu.edu.cn; sujing@jlu.edu.cn \\ 2020-06-30 收稿, 2020-08-18 修回, 2020-08-24 接受, 2020-08-25 网络版发表 \\ 国家自然科学基金(8167294，81772794)资助
}

\begin{abstract}
摘要目前认为, 关键激酶以及调控蛋白的折叠和降解失调与肿瘤的发生发展密切相关, 分子伴侣通过整合蛋白 质组 (proteome) 的折叠与降解机制在维持蛋白稳态中发挥了至关重要的作用. HSP90等伴侣蛋白的异常高表达被 认为是恶性肿瘤的标志, 通过参与细胞生存以及调亡途径等关键蛋白的调节, 为肿瘤提供了生存优势. HSP90一方 面与其他伴侣蛋白相互作用稳定“客户蛋白”的构象，另一方面还能够辅助蛋白酶体途径介导蛋白的降解．最近的 研究提示, HSP90的乙酰化等翻译后修饰是维持其功能的关键机制之一。组蛋白去乙酰化酶6(HDAC6) 是重要的自 噬受体，通过去乙酰化皮层肌动蛋白(cortactin)参与自噬体与溶酶体的融合.目前发现，HDAC6通过降低HSP90的 乙酰化水平维持了HSP90分子伴侣的功能, 保证客户蛋白的稳定折叠和成熟释放; 同时, HDAC6还作为 HSP90的客 户蛋白, 其活性也受到HSP90的调节. 因此, 进一步研究HSP90与HDAC6的交互作用可能为理解肿瘤细胞的分子伴 侣如何协调蛋白折叠与自噬降解机制维持自身生存提供新的思路. 本文总结了目前对HSP90以及HDAC6在蛋白折 叠以及降解机制中的研究进展, 提出HDAC6与HSP90抑制剂联合应用治疗肿瘤, 为靶向HSP90-HDAC6途径抑制肿 瘤增殖转移提供新的方向与思路.
\end{abstract}

关键词 组蛋白去乙酰化酶 6 , 热休克蛋白 90 , 蛋白质折叠与降解, 肿瘤增殖与转移

蛋白质的高效生产、折叠和分泌是肿瘤快速增殖 的保证. 为应对恶劣的环境, 肿瘤细胞往往通过上调多 种分子伴侣的表达来促进蛋白质的折叠 ${ }^{[1]}$. 然而, 新合 成的蛋白质中有近 $30 \%$ 的错误折叠蛋白, 这些蛋白将通 过泛素-蛋白酶体系统以及细胞自噬过程被降解，防止 形成毒性聚集体导致细胞死亡 ${ }^{[2]}$. 蛋白质折叠与降解 的相互协调是维持细胞内蛋白质量控制及稳定的关键 机制, 靶向蛋白质折叠和降解途径治疗癌症已成为研 究的焦点.

热休克蛋白90(heat shock protein 90, HSP90)作为
伴侣蛋白, 既是参与蛋白质折叠的重要分子伴侣, 又发 挥了促进蛋白降解的功能 ${ }^{[3]}$. 在恶性肿瘤中, HSP90作 为增殖转移的关键调节因子促进了多种致癌蛋白的成 熟与激活 ${ }^{[4]}$. HSP90结合辅助分子伴侣传递来的客户蛋 白形成复合体后进人折叠循环，经过一系列三磷酸腺 苷(ATP)水解供能及辅助分子伴侣的帮助，改变HSP90 蛋白 $\mathrm{N}$ 端的开闭状态, 最终达到促进客户蛋白的正确折 叠与成熟的目的. 此外, HSP90还可以携带客户蛋白与 蛋白酶体相结合, 并辅助客户蛋白的降解. HSP90的活 性受翻译后修饰的调控, 其中HSP90蛋白的乙酰化已被

引用格式: 田䜭, 颜晓羽, 于司杭, 等. HDAC6-HSP90途径介导蛋白折叠和降解调控肿瘤细胞增殖与转移. 科学通报, 2020, 65: 4223-4234 Tian R, Yan X Y, Yu S H, et al. HDAC6-HSP90 pathway-mediated protein folding and degradation regulates tumor cell proliferation and metastasis (in Chinese). Chin Sci Bull, 2020, 65: 4223-4234, doi: 10.1360/TB-2020-0644 
广泛研究. 一些研究表明, 沉默组蛋白去乙酰化酶 6 (Histone deacetylase 6, HDAC6)可以获得HSP90的乙酰 化产物, 从而减弱HSP90的伴侣功能, 降低客户蛋白的 稳定性.

HDAC6 是组蛋白去乙酰化酶(Histone deacetylase, $\mathrm{HDAC}$ )家族重要的成员，主要存在于细胞浆中，可作 为自噬受体(autophagy receptor)发挥作用. 而自噬(autophagy)则是细胞中维持内稳态的重要生命过程, 它通 过降解错误折叠的蛋白以及损伤的细胞器发挥细胞保 护作用. 此外, HDAC6自身还具有一定的独特性: 首先, HDAC6以自己为核心吸引泛素化蛋白成为蛋白聚集 体的支架，并通过去乙酰化皮层肌动蛋白(cortactin)参 与自噬体与溶酶体的融合. 除此之外, HDAC6的去乙 酰化作用还调节着HSP90的伴侣功能. 近年研究发现, HDAC6的去乙酰化酶特性及HSP90的伴侣功能为肿瘤 增殖转移创造了条件, 促进了相关癌蛋白的稳定, 并通 过参与自噬调节过程促进肿瘤细胞存活. 目前, 在膀胱 癌、肺癌、胃癌、乳腺癌、黑色素瘤、甲状腺等多种 肿瘤中, HSP90以及HDAC6异常高表达 ${ }^{[5 \sim 10]}$. 因此, HSP90-HDAC6可能通过参与蛋白质的折叠及降解机 制, 维持细胞内的蛋白质稳态, 为肿瘤的存活、增殖及 转移创造条件. 进一步探讨HSP90-HDAC6信号的作用
有望为肿瘤治疗提供新的靶点.

本文讨论了肿瘤细胞中HDAC6-HSP90相互作用 如何调控蛋白质的折叠与降解(图1), 以及HDAC6抑制 剂(HDAC6 inhibitor, HDAC6i)诱导HSP90乙酰化如何 调控肿瘤相关蛋白表达, 增强HSP90抑制剂与HSP90的 结合能力, 从而达到抑制肿瘤细胞的增殖与侵袭转移 的作用.

\section{HDAC6与HSP90的调控}

\subsection{HSP90调控蛋白折叠}

HSP90是一种ATP依赖的高度保守伴侣蛋白, 介导 多种肿瘤相关蛋白的折叠和修复 ${ }^{[11]}$. 根据亚细胞定位 的不同，目前将HSP90家族分为 4 类：定位于细胞质和 细胞核的HSP $90 \alpha$ 和HSP90 $\beta$ 、定位于内质网的葡萄糖 调节蛋白94(GRP94)以及主要分布在线粒体的肿瘤坏 死因子受体蛋白1(tumor necrosis factor receptor associated protein 1, TRAP1). 其中HSP90 $\alpha$ 是可诱导形式, 而HSP90 $\beta$ 是组成性表达形式, 两者均形成二聚体, 并且 在结构和功能方面显著相似 ${ }^{[12 \sim 14]}$. 在结构上, 家庭成员 由结合ATP的N-末端结构域、包含亚细胞定位序列的 C-末端结构域以及中间结构域组成. 其中, 当HSP90缺

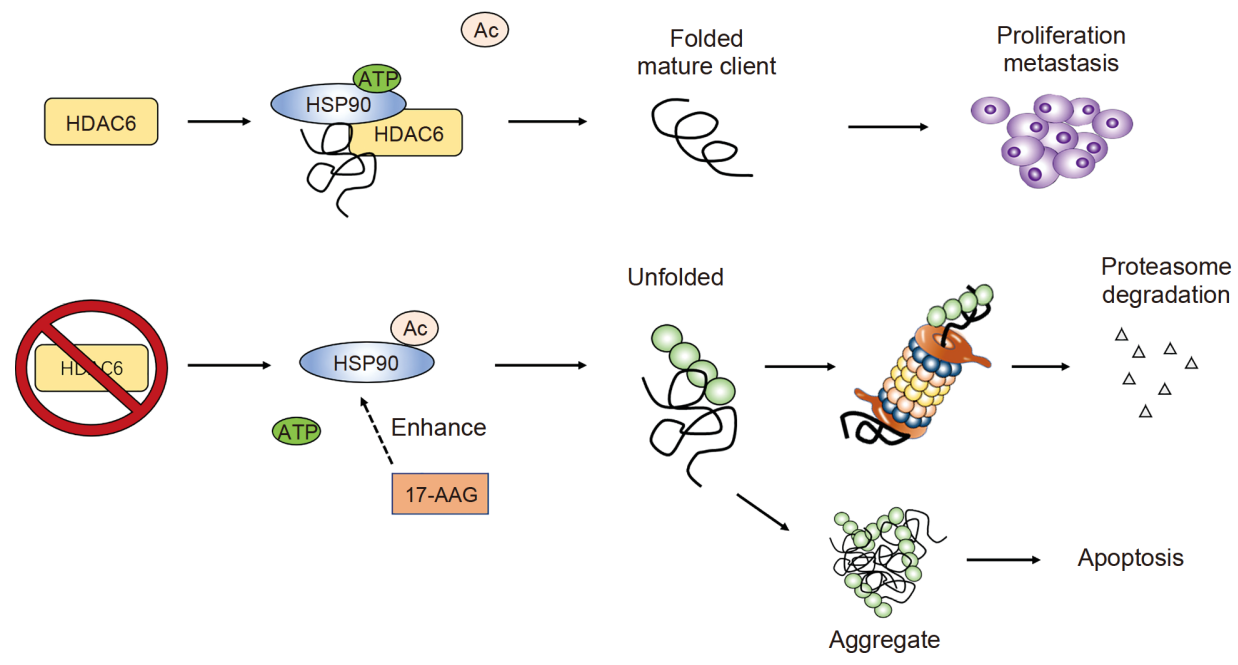

图 1 (网络版彩色)HDAC6参与HSP90的调控. HDAC6抑制HSP90的乙酰化水平, 促进HSP90与客户蛋白的结合, 从而调控细胞增殖转移相关 途径蛋白的折叠; 当抑制HDAC6时, HSP90过度乙酰化, 增强了HSP90与其抑制剂17-AAG(tanespimycin)的相互作用, 减弱了HSP90与ATP结合 的能力, 从而影响HSP90的蛋白折叠. 一部分错误折叠的蛋白经蛋白酶体途径降解清除, 而另一部分蛋白因过度堆积形成毒性聚集体导致细胞 调亡

Figure 1 (Color online) HDAC6 participates in the regulation of HSP90. HDAC6 inhibits the acetylation level of HSP90 and promotes the binding of client proteins to HSP90, thereby regulating the folding of proteins related to cell proliferation and metastasis; when HDAC6 is inhibited, HSP90 is over-acetylated, which enhances the interaction between HSP90 and its inhibitor 17-AAG. It weakens the ability of HSP90 to bind to ATP, thereby affecting the protein folding of HSP90. Part of the misfolded proteins are degraded and eliminated by the proteasome pathway, while the other part of the proteins are excessively accumulated to form toxic aggregates and cause apoptosis 
乏C-末端结构域时，其ATP酶活性会受到损害; 中间结 构对HSP90与客户蛋白的相互作用、自身功能和结构 的灵活性至关重要 ${ }^{[11,15 ~ 19]}$.

研究表明, HSP90能够完成如此复杂的调控过程还 依赖一些辅助分子伴侣，如HSP40、HSP70、P23、 $\mathrm{CHOP}(\mathrm{C} / \mathrm{EBP}-$ homologous protein)等的帮助. 首先, 辅 助分子伴侣HSP40与客户蛋白质结合产生的相互作用 导致HSP70蛋白的募集，HSP70在此阶段仅与HSP40较 弱结合. HSP70/HSP40复合物的形成触发ATP水解, 引 发HSP70的构象变化, 增加其与底物的亲和力 ${ }^{[20,21]}$. 其 次, HSP70与HSP90的相互作用还需要Hop(应激诱导蛋 白1，Sti1)的参与，二磷酸腺苷(adenosine diphosphate, ADP)结合的HSP70可以与Hop相互作用，同时Hop也在 携带客户蛋白的HSP70/HSP40复合物与HSP90二聚体 之间起到了重要的衔接作用 ${ }^{[22,23]}$. 随后, HSP90伴侣复
合物与ATP结合，迫使HSP90呈现“封闭”构象，p23通过 稳定闭合HSP90二聚体构象起到减慢ATP水解的作用, 从而减弱 HSP90的功能活性. 最后, ATP水解为ADP导 致HSP90再一次变为开放构象并释放成熟的客户蛋白

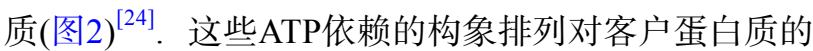
正确折叠和随后的稳定性至关重要.

研究发现，HSP90通过调控客户蛋白折叠参与肿 瘤细胞增殖、调亡以及血管生成等多条信号 ${ }^{[25]}$. HSP90维持生长因子受体生存信号激酶，如AKT、磷 脂肽肌醇-3-激酶(lecithin exception inositol-3-kinase, $\mathrm{PI} 3 \mathrm{~K})$, 以及癌基因的稳定 ${ }^{[26,27]}$. 在伯基特(Burkitt)淋巴 瘤中, HSP90抑制剂PU-H71处理24 h后, PI3K下游的4E 结合蛋白1(4E-binding protein 1,4EBP1)蛋白表达显著 降低，表明HSP90参与了PI3K途径关键蛋白的调控 ${ }^{[28]}$. 应激状态下HSP90-AKT与促凋亡激酶ASK1(apoptpsis

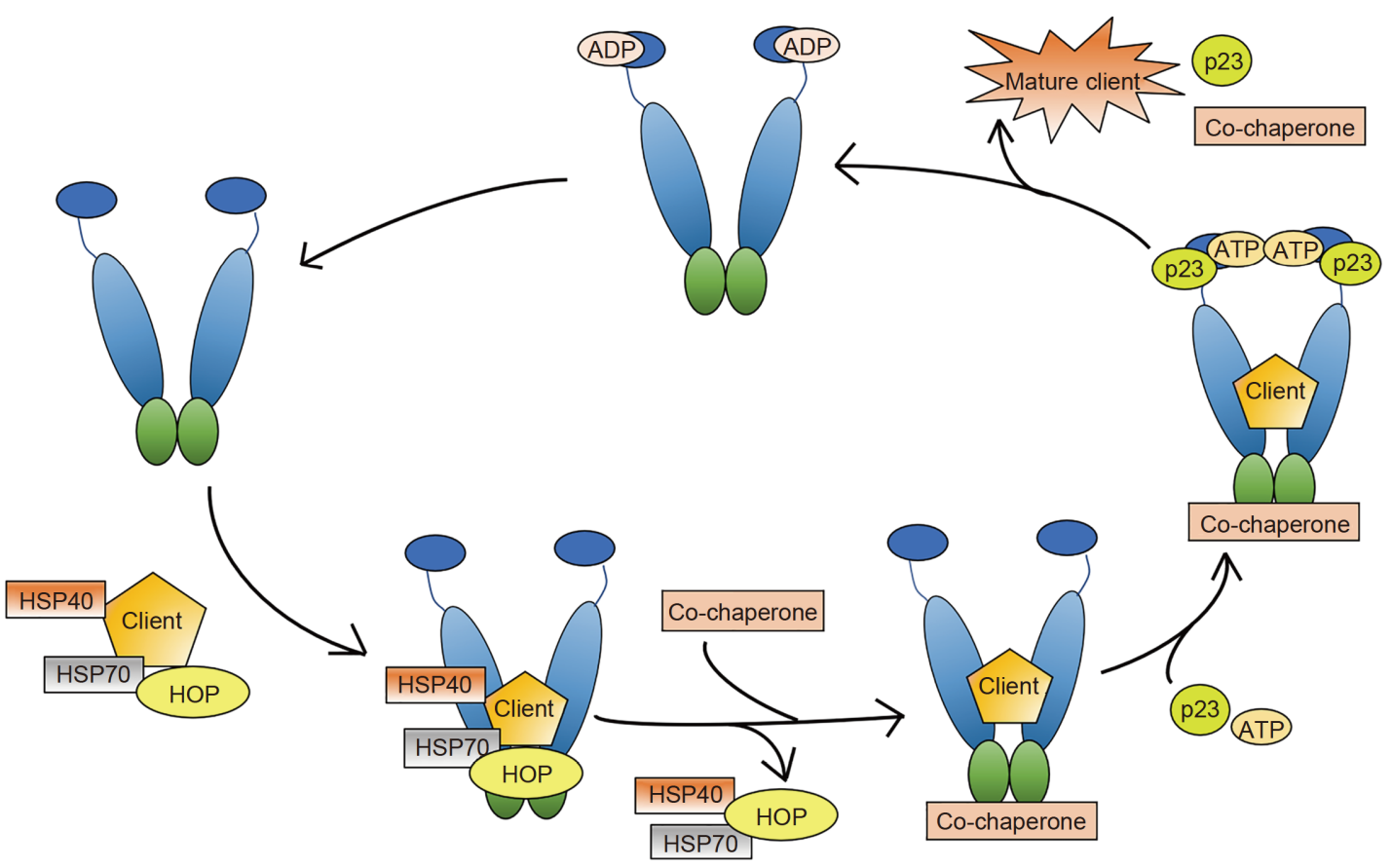

图 2 (网络版彩色)HSP90协助客户蛋白质折叠的机制. 首先，伴侣蛋白HSP40与客户蛋白结合产生的相互作用募集了HSP70蛋白，HSP70/ HSP40复合物由此形成并触发ATP水解, 这引发了HSP70的构象变化并且增强了HSP70与底物的亲合力. 其次, 与ADP结合的HSP70可以与Hop 相互作用，通过Hop的衔接作用与HSP90结合，将客户蛋白成功运送至HSP90. 此时，HSP90与ATP结合再次引起自身构象变化，HSP90呈现“封 闭”构象, 辅助分子伴侣p 23 通过稳定HSP 90 的闭合构象, 达到减缓ATP水解的目的. 最后, 当ATP又一次水解为ADP导致HSP90变为开放构象, 成 熟的客户蛋白质也由此释放

Figure 2 (Color online) The mechanism of HSP90 assists client protein folding. Firstly, the interaction between the chaperone protein HSP40 and the client protein recruits the HSP70 protein, and the HSP70/HSP40 complex forms and triggers ATP hydrolysis, which triggers a conformational change of HSP70 and enhances the affinity of HSP70 to the substrate. Secondly, HSP70 that binds to ADP can interact with Hop, and bind to HSP90 through Hop's cohesion to successfully deliver client protein to HSP90. At this time, the combination of HSP90 and ATP again causes HSP90's own conformational change, HSP90 assumes a closed conformation, and the auxiliary molecular chaperone p23 achieves the purpose of slowing down the hydrolysis of ATP by stably closing the conformation of HSP90. Finally, when ATP is hydrolyzed to ADP again, HSP90 becomes an open conformation, then mature client proteins are released 
signal regulating kinase 1)形成的复合物抑制了ASK1的 促调亡活性；而当抑制HSP90时，该复合物(HSP90AKT-ASK1)的构象则会发生变化, ASK1的促凋亡活 性被重新激活, 由此增强了应激物(如 $\left.\mathrm{H}_{2} \mathrm{O}_{2}\right)$ 诱导的细 胞调亡. HSP90还可以通过结合血管内皮生长因子 (vascular endothelial growth factor, VEGF), 激活一氧 化氮合酶(nitric oxide synthase, NOS)和基质金属蛋白 酶2(matrix metalloproteinase 2, MMP2)促进血管的生 成和转移 ${ }^{[11]}$. VEGF刺激内皮细胞将 $\mathrm{NOS}$ 和AKT募集 到HSP90结构上, 促进eNOS磷酸化、酶的激活以及 NO的释放 ${ }^{[29]}$. 线粒体中, HSP90与TRAP1(TNF receptor associated protein 1)、CYLD(cyclophilin D)和survivin 相互作用, 发挥抗调亡功能. 研究表明, 用HSP90抑制 剂GA(geldanamycin)处理HeLa细胞后, HSP90伴侣功 能受到抑制，客户蛋白被蛋白酶体降解， survivin的表 达随GA浓度的变化呈现依赖性损失, 并因此诱导了线 粒体凋亡的激活 ${ }^{[30]}$. 据报道, 在氧化过程中由亚基环组 成的 $20 \mathrm{~S}$ 蛋白酶体蛋白复合物通过结合HSP90, 选择性 识别和降解客户蛋白. 氧化的钙调蛋白 (calmodulin, $\mathrm{CaM}$ )仅在HSP90存在的情况下才会被20S蛋白酶体显 著降解(表1). 以上研究提示, HSP90可能为肿瘤治疗的
潜在靶点.

\subsection{HDAC6通过翻译后修饰调控HSP90参与蛋白 降解}

研究发现, HSP90过度乙酰化会导致其活性受到抑 制, 直接造成客户蛋白折叠异常. 在胞浆中发挥作用的 组蛋白去乙酰化酶HDAC6在维持HSP90的功能和活性 中发挥了重要作用. HDAC6 是HDAC家族中唯一具有 两个串联的含有催化活性的去乙酰化酶结构域(DD1、 DD2)的成员，具有独特的结构特征. HDAC6属于 $\mathrm{Zn}^{2+}$ 依赖性蛋白酶, 可以在细胞质与细胞核之间穿梭. 核输出信号(nuclear export-signal, NES)与含有色氨酸谷氨酰胺的十四肽确保将HDAC6针定在细胞质中, 保 证细胞质中HDAC6的稳定表达. C末端含有的锌指结 构域(ZnF-UBP)可与单链或多链泛素相结合形成聚集 体，HDAC6可以通过该结构域募集泛素化蛋白促进自 噬进程(图3).

HDAC6通过调控HSP90的乙酰化水平影响HSP90 客户蛋白的表达. 研究表明, 在缺乏HDAC6的小鼠胚胎 成纤维细胞中乙酰化的HSP90水平升高, 这些细胞中的 糖皮质激素受体功能受损, 作为HSP90客户蛋白的雄激

\section{表 1 肿瘤相关的HSP90客户蛋白}

Table 1 Cancer-related client proteins of HSP90

\begin{tabular}{|c|c|c|c|c|}
\hline HSP90客户蛋白 & 作用位点 & 分子功能 & 肿瘤中的功能 & 文献 \\
\hline VEGF受体 & 细胞膜 & 血管生成 & 新血管形成 & \multirow{3}{*}[31]{} \\
\hline AKT(PDK1、GSK3 $\beta 、 \mathrm{mTOR})$ & 细胞质 & 通过PI3K通路调节细胞存活信号 & 抗调亡细胞存活信号 & \\
\hline DAPK(death associated protein kinase) & 细胞质 & 介导多种细胞死亡相关的过程 & 肿瘤抑制 & \\
\hline ASK1 & 细胞质 & 诱导细胞凋亡、细胞增殖、分化和存活 & $\begin{array}{l}\text { HSP90-Akt-ASK1形成复合物, 抑制 } \\
\text { 促调亡活性 }\end{array}$ & {$[32]$} \\
\hline CYLD & 线粒体 & 介导氧化应激条件下异常蛋白的降解 & 肿瘤抑制 & \multirow{2}{*}{ [33] } \\
\hline Survivin & 线粒体 & 抑制细胞凋亡 & 促进肿瘤的增殖、分化和侵袭 & \\
\hline 20S蛋白酶体 & 细胞质 & 介导氧化应激条件下异常蛋白的降解 & $\begin{array}{c}\text { HSP90增强了20S蛋白酶体对氧化钙 } \\
\text { 调蛋白的降解 }\end{array}$ & [34] \\
\hline
\end{tabular}

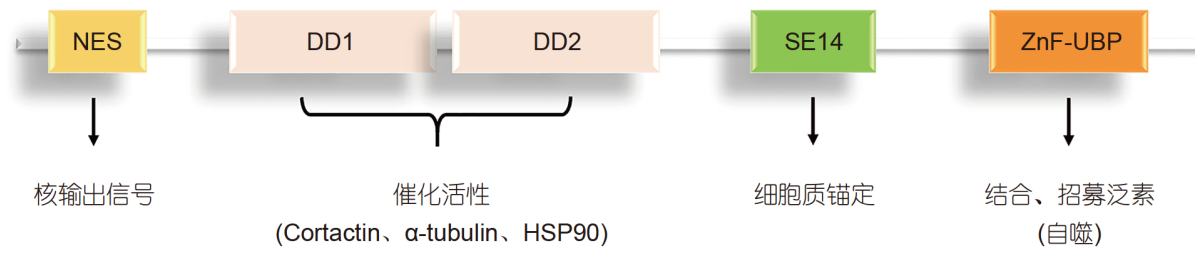

图 3 (网络版彩色)HDAC6的功能性结构域

Figure 3 (Color online) The functional domains of HDAC6 
素受体也在HDAC6抑制后蛋白表达下调 ${ }^{[35,36]}$. HDAC6 表达的减少还影响了另一种HSP90客户蛋白HIF- $\alpha$ (hypoxia inducible factor-1)的稳定性 ${ }^{[37]}$. 癌症相关信号通 路EGFR-RAS-RAF-MEK诱导HDAC6在Ser1035位的 磷酸化，当抑制HDAC6阻断这一途径促使白血病细胞 和肺癌细胞中的HSP90乙酰化, 并抑制HSP90与ATP结 合, 使其与客户蛋白的相互作用丧失或减弱 ${ }^{[38,39]}$. 研究 表明，HDAC6介导的HSP90去乙酰化可以结合并保护 这些癌蛋白免受蛋白酶体的降解 ${ }^{[40]}$. 同时，HDAC6 可 以调节HSP90介导的VEGF受体的稳定，血管内皮生长 因子受体(vascular endothelial growth factor receptor, VEGFR) 在肿瘤生长和血管生成中起关键作用, HDAC6的敲除会导致HSP90的客户蛋白VEGFR降 解 ${ }^{[41]}$. 癌症相关的转录因子 $\beta$-cantenin也与HDAC6 和 HSP90相关. PKC $\alpha$ (protein kinase C $\alpha$ )通过磷酸化 HDAC6增强HDAC6去乙酰化 $\beta$-cantenin的活性， $\beta$-cantenin的去乙酰化支持其核转位与抗病毒基因表达 ${ }^{[42]}$. 有证据证明, HSP90活性在维持PKC $\alpha$ 磷酸化中起着重 要作用, HSP90保护PKCa不受HSP70/HSC70依赖性蛋 白酶体途径参与的降解 ${ }^{[43]}$. 此外, HSP90有助于LFP、 突变体AKT、c-KIT(v-kit Hardy-Zuckerman 4 feline sarcoma viral oncogene homolog)、白血病标记蛋白 WT1(Wilms tumor protein)和P53突变体的折叠与稳定 性 ${ }^{[44 \sim 47]}$. 有关白血病细胞的研究表明, HDAC6调节 HSP90的乙酰化从而调节BCR-ABL融合蛋白和FLT3ITD的蛋白酶体降解 ${ }^{[48]}$. 在急性髓细胞白血病 (acute myeloid leukemia, AML)中, G蛋白偶联受体激酶 $2(\mathrm{G}$ protein-coupled receptor kinases 2, GRK2)与HSP90相 关, 而GRK2可磷酸化HDAC6从而激活 $\alpha$-tubulin ${ }^{[49]}$. 由 此可见, HDAC6与HSP90之间的翻译后修饰关系影响 着癌症的发生发展, 肿瘤的增殖、侵袭转移, 进而有可 能导致癌症药物的耐受.

\section{HDAC6-HSP90调控自噬途径}

自噬过程是动态变化的, 通过双层膜结构包裹待 降解的蛋白形成自噬体, 将自噬底物运送向溶酶体, 在 溶酶体内完成降解, 分解出的大分子物质最终释放回 细胞质, 而这一系列动态的过程也被称为自噬流( $\mathrm{au}-$ tophagy flux). 在该过程中HDAC6作为自噬受体, 帮助 泛素化蛋白转运至自噬体降解. 同时, HDAC6还能够通 过其去乙酰化酶活性调控自噬体的运动 ${ }^{[47,50]}$. 研究表 明，当蛋白酶体功能受到抑制时，HDAC6通过与泛素
结合来感知错误折叠的泛素化蛋白，同时激酶ERK1 (extracellular-regulated kinase 1)通过磷酸化HDAC6, 增强 $\alpha$-微管蛋白的乙酰化来维持微管的稳定性并促进 细胞的转移 ${ }^{[39]}$. 随后, HDAC6 通过与动力蛋白的相互 作用将结合的泛素蛋白沿着微管中心逆行运输，最终 促进泛素化蛋白积累形成聚集体 ${ }^{[51,52]}$. 蛋白酶体受抑 制时, 通过去乙酰化cortactin引起F-actin聚集形成肌动 蛋白网络，HDAC6促进自噬小体与溶酶体的融合从而 调节自噬成熟(图4) $)^{[53]}$. 有研究表明, 自噬促进了细胞 侵袭转移. 研究结果提示, 自噬促进了基质金属蛋白酶 MMP2在肝细胞癌(hepatocellular carcinoma, HCC)细胞 中的表达，从而促进了细胞外基质(extracellular matrix, $\mathrm{ECM})$ 的降解, 增强了 $\mathrm{HCC}$ 细胞的转移能力 ${ }^{[54]}$. 当抑制 HDAC6时, 自噬流量受阻导致自噬相关蛋白p62积累, 通过活化caspas8启动细胞凋亡信号 ${ }^{[55]}$. 过度积累的p62 可以通过与致癌蛋白Twist1相结合, 稳定 Twist1蛋白的 表达阻止其降解，进而达到促进肿瘤细胞增殖与转移 的目的 ${ }^{[5]}$. 以上证据证明, HDAC6 可能通过细胞自噬 参与了肿瘤细胞存活、增殖以及转移机制的调控.

尽管自噬具有双重作用, 但多数研究认为, 通畅的 自噬流可能作为一种保护机制促进肿瘤细胞的存 活 ${ }^{[57 ~ 59]}$. 我们实验组发现, 索拉非尼能够增加肝癌细胞 的自噬水平，同时能够通过上调HDAC6去乙酰化酶活 性促进HSP90的折叠功能, 导致HSP90的客户蛋白 PKM2(M2型丙酮酸激酶)的活性增强, 降低了HCC 细胞 对索拉非尼的敏感性(工作未发表)。值得注意的是，近 年来的研究发现, HDAC6与自噬相关蛋白p62的交互作 用对自噬流的调节至关重要. 自噬相关蛋白p62可能参 与了HDAC6去乙酰化酶活性的调节. 在前列腺癌中, p62的过表达导致HDAC6蛋白水平的增加，酶活性增 强, $\alpha$-微管蛋白的乙酰化和微管稳定性降低, 促进了前 列腺癌细胞发生上皮-间质的转化; 然而, HDAC6携带 泛素化蛋白通过微管运输的途径受到阻碍，这不利于 自噬体完成降解过程 ${ }^{[60]}$. 另一方面, 研究发现, p62通过 与HDAC6酶催化结构域DD2相互作用，抑制了HDAC6 的酶活性 ${ }^{[61]}$. 在小鼠胚胎成纤维细胞中抑制p62的表达 能够增强HDAC6的去乙酰化酶活性, 导致cortactin的募 集急剧增加, 但p62缺失抑制了F-actin的重塑, 进而抑制 了自噬小体与溶酶体的融合, 阻断了自噬流.

最近的研究发现, HSP90也参与了细胞自噬中多个 关键分子的调控. 例如，自噬抑制途径AKT/mTOR (mammalian target of rapamycin)中的AKT蛋白、自噬 


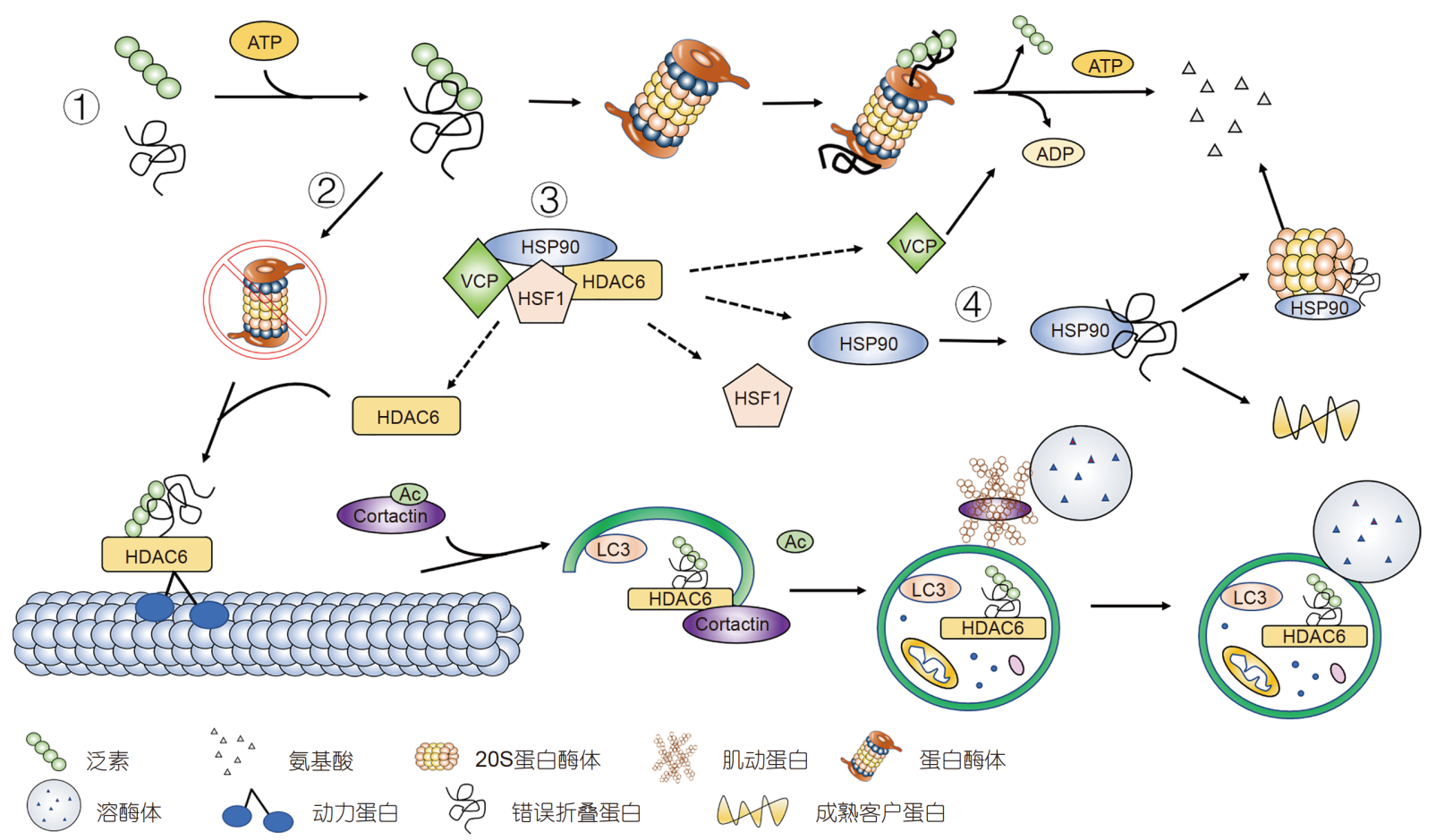

图 4 (网络版彩色)HDAC6与HSP90参与肿瘤细胞蛋白质折叠及降解. (1) 细胞中错误折叠的蛋白经蛋白酶体途径降解. (2) 一些不能被蛋白酶 体清除的大分子蛋白, 被HDAC6募集经自噬途径降解. HDAC6还能通过降低cortactin的乙酰化水平促进溶酶体与自噬体融合. (3) 当HDAC6识 别错误折叠蛋白并与其结合, 复合物(VCP-HSF1-HSP90-HDAC6)开始解离, HSF1 由此释放, 诱导热休克因子与HSP90转录促进细胞存活. (4) HSP90帮助20s蛋白酶体选择性识别HSP90客户蛋白, 并促进客户蛋白降解

Figure 4 (Color online) HDAC6 and HSP90 are involved in protein folding and degradation of tumor cells. (1) Misfolded proteins in cells are degraded by the proteasome pathway. (2) Some macromolecular proteins that cannot be cleared by the proteasome are recruited by HDAC6 and degraded by the autophagy pathway. HDAC6 can also promote the fusion of lysosomes and autophagosomes by reducing the acetylation level of cortactin. (3) When HDAC6 recognizes the misfolded protein and binds to it, the complex (VCP-HSF1-HSP90-HDAC6) begins to dissociate, and HSF1 is released, which induces heat shock factor and HSP90 transcription to promote cell survival. (4) HSP90 helps 20s proteasome to selectively recognize HSP90 client protein and promote client protein degradation

相关蛋白Beclin1、Ulk1(unc-51 like autophagy activating kinase 1)以及LAMP2A(lysossomal associated protein $2 \mathrm{~A})$ 等均作为HSP90的客户蛋白, 受HSP90的调控 ${ }^{[62 \sim 65]}$. 过表达HSP90AA1显著降低了AKT和mTOR的磷酸化 水平, 导致PI $3 \mathrm{~K} / \mathrm{AKT} / \mathrm{mTOR}$ 途径受到抑制, 进而上调 细胞自噬, 导致骨肉瘤细胞产生化疗耐受 ${ }^{[66]}$. 非小细胞 肺癌中, HSP90抑制剂ganetespib通过下调HSP90的非 典型客户蛋白ATG7(autophagy-related gene 7)的表达 抑制自噬 ${ }^{[67]}$. 另外, HDAC6-HSP90-VCP-HSF1复合体 也参与了自噬的调控. 当HDAC6识别错误折叠蛋白与 其结合时, 该复合物解离, 解离出的 $\mathrm{VCP}$ (valosin-containing protein)不仅是错误折叠蛋白降解的调节剂, 同 时VCP利用其ATP酶活性解离HSP90-HSF1复合物, 释 放的HSF1(热休克因子)诱导热休克因子与HSP90转录, 促进客户蛋白折叠并激活HSP基因促进细胞存活(图 $4)^{[47,52,68]}$. 以上研究提示, 探讨HDAC6-HSP90之间的交
互作用可能成为靶向自噬治疗肿瘤的潜在途径，有望 帮助我们更全面地理解蛋白降解途径在肿瘤细胞存 活、增殖以及转移中的作用.

\section{HDAC6-HSP90介导肿瘤增殖转移}

\subsection{HDAC6-HSP90对增殖转移相关靶蛋白的 调控}

目前研究认为, HDAC6与HSP90均在肿瘤增殖和 转移信号中发挥了关键作用. 首先, HDAC6通过去乙 酰化丝裂原活化蛋白激酶(mitogen-activated protein kinase, MAPK)信号转导途径下游激酶ERK1, 促进ERK1 的激酶活性 ${ }^{[69]}$. 此外, HDAC6还能调节表皮生长因子 受体(epidermal growth factor receptor, EGFR)信号, HDAC6通过对 $\alpha$-微管蛋白的去乙酰化作用控制EGFR 的运输和降解 ${ }^{[70]}$. HDAC6 缺失, 促进乙酰化 $\alpha$-微管蛋白 
积累, 导致依赖微管的蛋白运输能力增强, 加速了 EGFR的降解 ${ }^{[71 ~ 73]}$. 另一方面, HSP90通过维持AKT的 磷酸化水平, 上调PI3K/AKT途径促进肿瘤细胞的存活 和转移 ${ }^{[74,75]}$. 同时HSP90抑制剂AUY922可以通过下调 EGFR、Met(mesenchymal to epithelial transition factor) 和AXL(AXL receptor tyrosine kinase), 破坏AKT信号, 抑制肺癌细胞增殖, 诱导细胞凋亡 ${ }^{[76]}$.

以上只是HDAC6与HSP90自身对EGFR、ERK和 $\mathrm{AKT}$ 等增殖相关蛋白的调控. 最近的研究发现, HDAC6与HSP90的相互作用也参与了肿瘤增殖转移关 键蛋白的调控. Tao等人 ${ }^{[77]}$ 的实验表明, HDAC6 通过抑 制HSP90的乙酰化水平，促进客户蛋白EGFR活性，激 活下游AKT与ERK通路，从而促进了肿瘤细胞的增殖. 此外，HDAC6还可以通过去乙酰化HSP90稳定促肿瘤 细胞存活的巨噬细胞迁移抑制因子 (macrophage migration inhibitory factor, MIF)的表达. Schulz等人 ${ }^{[78]}$ 在研究 中表明，当抑制HDAC6时HSP90的过度乙酰化会严重 破坏MIF蛋白，从而达到抑制肿瘤生长的目的. 另一方 面, 在乳腺癌中, 抑制HSP90促进了HDAC6的降解, 导 致微管蛋白的过度乙酰化, 进而抑制了乳腺癌细胞的 转移(表2). 因此, HDAC6-HSP90途径可能涉及多个肿 瘤增殖转移相关靶点的调控，而靶向HDAC6-HSP90的 药物也成为近年来的研究热点.

\section{2 靶向HDAC6-HSP90抑制肿瘤增殖转移的药物}

目前已报道了多种具有不同帽基团(cap group)、 接头和锌离子结合基团(zinc binding group, ZBG)的 HDAC6 抑制剂 ${ }^{[84]}$. HDAC6抑制剂虽然可以通过阻断
自噬流促进了肿瘤细胞的药物敏感性，但同时过度乙 酰化的微管蛋白也促进了肿瘤细胞的侵袭转移. 因此 针对HDAC6抑制剂的双重作用，越来越多的研究倾向 于将其他药物同HDAC6抑制剂的联合应用，提升药物 治疗效果. HSP90抑制剂主要靶向分子伴侣N木端的 ATP结构域，也有少部分抑制剂是针对蛋白质的C端结 构域，但由于 $\mathrm{C}$ 端缺乏明确的配体结合口袋，限制了这 类抑制剂的效果 ${ }^{[85]}$.

随着HDAC6与HSP90之间相互作用的深人研究, HDAC6抑制剂与HSP90抑制剂的联合应用成为了癌症 治疗中更为有效的新突破点. GA及其衍生物(如17AAG、Alvespimycin)相比于核苷酸以更高的亲和力与 $\mathrm{ATP} / \mathrm{ADP}$ 结合口袋相结合，从而抑制了HSP90与客户 蛋白的结合能力. 同时有研究表明，抑制HDAC6可增 强HSP90与 $17-A A G$ 的结合, HSP90高度乙酰化使ATP 与HSP90的结合减弱，导致HSP90与AR(androgen receptor)、GR(glucocorticoid receptor)和ER $\alpha$ (estrogen receptor)及其他相关癌蛋白激酶，如Bar-Abl、FLT-3 (FMS-like tyrosine kinase-3)、AKT和HER-2(表皮生长 因子受体)等客户蛋白的结合被破坏 ${ }^{[86-89]}$. 在白血病细 胞中, HSP90抑制剂17-AAG联合HDAC6抑制剂，通过 促进HSP90高度乙酰化, 更有效地抑制HSP90与客户蛋 白的结合，从而达到抑制肿瘤细胞增殖转移的目的(表 2). 抑制HDAC6可以破坏HSP90与客户蛋白的结合能 力, 抑制食管鳞状细胞癌(esophageal squamous carcino$\mathrm{ma}, \mathrm{ESCC}$ )的增殖和转移. 在ER $\alpha$ 高表达的乳腺癌细胞 系T47D中，HDAC6与HSP90抑制剂的联用导致ER $\alpha$ 的 表达显著降低(表2)。尽管人们对HDAC6与HSP90抑制

\section{表 2 HDAC6与HSP90抑制剂联用治疗肿瘤}

Table 2 HDAC6 inhibitors combined with HSP90 inhibitors for tumor treatment

\begin{tabular}{|c|c|c|c|c|}
\hline \multirow{2}{*}{ 癌症 } & \multicolumn{2}{|c|}{ 联合应用 } & \multirow{2}{*}{ 相关通路 } & \multirow{2}{*}{ 文献 } \\
\hline & HDAC6抑制剂 & HSP90抑制剂 & & \\
\hline 乳腺癌 & Tubacin & 17-DMAG & 抑制微管蛋白去乙酰化, 增强微管稳定性 & [79] \\
\hline 尿路上皮癌 & Tubacin & 17-AAG & 减少ERK磷酸化 & [80] \\
\hline 白血病 & Tubacin & 17-AAG & $\begin{array}{c}\text { HSP90的高乙酰化可以使 } 17-A A G \text { 更有效地抑制HSP90与 } \\
\text { p23、ATP和客户蛋白的结合 }\end{array}$ & [81] \\
\hline \multirow{4}{*}{ 甲状腺癌 } & PXD101 & NVP-AUY922 & Survivin的失活和DNA损伤相关蛋白的活化 & [82] \\
\hline & PXD101 & SNX5422 & & \\
\hline & SAHA & SNX5422 & PI3K/AKT/mTOR信号通路 & [83] \\
\hline & TSA & SNX5422 & & \\
\hline 食管癌 & Tubastatin A & PU-H71 & $\begin{array}{l}\text { PI3K/AKT信号通路 } \\
\text { MAPK/ERK信号通路 }\end{array}$ & [77] \\
\hline
\end{tabular}


剂联合应用治疗肿瘤有了一定认识(表2), 但还存在许 多问题. 目前已经发现了许多癌症相关蛋白受到 HSP90的调控，例如AKT、c-Raf(Ras-associated factor1)、HER-2等，这为HSP90抑制剂在肿瘤中的应用提供 了理论依据. 但HSP90作用范围的广泛性导致其在癌细 胞中发挥的作用远比预期的更为复杂，可能引起“脱靶

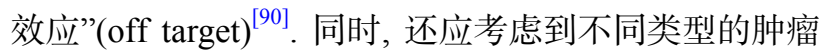
对于HSP90抑制剂的敏感性是否存在差异? 如何降低 抑制剂对于正常细胞的毒性作用? 解决以上问题为 HDAC6-HSP90抑制剂的临床应用以及肿瘤的精准治 疗提供理论依据.

\section{4 讨论}

靶向HDAC6-HSP90调控蛋白折叠和降解途径为 抑制肿瘤的增殖和转移拓展了思路. 蛋白质的折叠与 降解维持了癌细胞各种功能蛋白质在细胞内的正常运 转, 为肿瘤的生存发展提供适宜条件. 通过抑制HDAC6 促进HSP90过度乙酰化，可有效地抑制HSP90与ATP之 间的结合, 影响HSP90对肿瘤增殖、转移相关蛋白的折
叠和降解 ${ }^{[44]}$. 同时, HDAC抑制剂可以帮助HSP90与其 抑制剂更加紧密地结合. 目前, 无论是单独抑制HDAC6 还是单独抑制HSP90, 在癌症治疗方面都存在不足. HDAC6的抑制会导致微管蛋白(tubulin)的高度乙酰化， 增加原发性乳腺肿瘤细胞的侵袭转移. 此外, 在2型神 经纤维瘤(neurofibromatosis type 2, NF2)细胞中, 微管 的去乙酰化会损害Hippo信号通路并影响细胞的接触 抑制。而单独抑制HDAC6还可能增强调节性T细胞功 能，从而导致肿瘤发生免疫逃逸. 与之类似，HSP90抑 制剂可以通过调控AKT/mTOR信号通路诱导自噬，进 而触发细胞保护机制. 因此，HDAC6-HSP90双靶点抑 制, 一方面能够减少自噬等代偿途径的活化, 另一方面 减弱了HSP90抑制剂可能造成的脱靶效应. 毫无疑问, HDAC6-HSP90双靶点的肿瘤治疗还有很长的路要走, 今后随着HDAC6、HSP90抑制剂在不同肿瘤细胞中研 究的深人, 弄清楚HDAC6-HSP90与自噬之间复杂的网 络关系，有助于人们进一步了解蛋白折叠与降解途径 在肿瘤细胞增殖以及转移中的作用，为增加肿瘤的治 疗效果提供新的靶点.

\section{参考文献}

1 Hartl F U, Hayer-Hartl M. Converging concepts of protein folding in vitro and in vivo. Nat Struct Mol Biol, 2009, 16: 574-581

2 Ellis R J. Protein misassembly: Macromolecular crowding and molecular chaperones. Adv Exp Med Biol, 2007, 594: 1-13

3 McClellan A J, Tam S, Kaganovich D, et al. Protein quality control: Chaperones culling corrupt conformations. Nat Cell Biol, 2005, 7: 736-741

4 Khurana N, Bhattacharyya S. Hsp90, the concertmaster: Tuning transcription. Front Oncol, 2015, 5: 100

5 Lettini G, Pietrafesa M, Lepore S, et al. Heat shock proteins in thyroid malignancies: Potential therapeutic targets for poorly-differentiated and anaplastic tumours? Mol Cell Endocrinol, 2020, 502: 110676

6 Liu K, Chen J, Yang F, et al. BJ-B11, an Hsp90 inhibitor, constrains the proliferation and invasion of breast cancer cells. Front Oncol, 2019, 9: 1447

7 Lee H W, Kim K M. Clinical significance of heat shock protein $90 \alpha$ expression as a biomarker of prognosis in patients with gastric cancer. Niger J Clin Pract, 2019, 22: 1698-1705

8 Zhang X, Yuan Z, Zhang Y, et al. HDAC6 modulates cell motility by altering the acetylation level of cortactin. Mol Cell, 2007, 27: 197-213

9 Hao M, Song F, Du X, et al. Advances in targeted therapy for unresectable melanoma: New drugs and combinations. Cancer Lett, 2015, 359: 1-8

10 Basu R, Wu S, Kopchick J J. Targeting growth hormone receptor in human melanoma cells attenuates tumor progression and epithelial mesenchymal transition via suppression of multiple oncogenic pathways. Oncotarget, 2017, 8: 21579-21598

11 Whitesell L, Lindquist S L. HSP90 and the chaperoning of cancer. Nat Rev Cancer, 2005, 5: 761-772

12 Ciocca D R, Arrigo A P, Calderwood S K. Heat shock proteins and heat shock factor 1 in carcinogenesis and tumor development: An update. Arch Toxicol, 2013, 87: 19-48

13 Buchner J, Li J. Structure, function and regulation of the Hsp90 machinery. Biomed J, 2013, 36: 106-117

14 Langer T. Intracellular localization of the $90 \mathrm{kDa}$ heat shock protein (HSP90 $\alpha$ ) determined by expression of a EGFP-HSP90 $\alpha$-fusion protein in unstressed and heat stressed 3T3 cells. Cell Biol Int, 2003, 27: 47-52

15 Jackson S E. Hsp90: Structure and function. Top Curr Chem, 2013, 328: 155-240

16 Lavery L A, Partridge J R, Ramelot T A, et al. Structural asymmetry in the closed state of mitochondrial Hsp90 (TRAP1) supports a two-step ATP hydrolysis mechanism. Mol Cell, 2014, 53: 330-343

17 Subbarao Sreedhar A, Kalmár É, Csermely P, et al. Hsp90 isoforms: Functions, expression and clinical importance. FEBS Lett, 2004, 562: 11-15

18 Csermely P, Schnaider T, Söti C, et al. The 90-kDa molecular chaperone family. Pharmacol Therapeutics, 1998, 79: 129-168

19 Dutta Gupta S, Bommaka M K, Banerjee A. Inhibiting protein-protein interactions of Hsp90 as a novel approach for targeting cancer. Eur J 
Medicinal Chem, 2019, 178: 48-63

20 Walter S, Buchner J. Molecular chaperones-Cellular machines for protein folding. Angew Chem Int Ed, 2002, 41: 1098-1113

21 Chaudhury S, Welch T, Blagg B. Hsp90 as a target for drug development. ChemMedChem, 2006, 1: 1331-1340

22 Murphy P J M, Kanelakis K C, Galigniana M D, et al. Stoichiometry, abundance, and functional significance of the Hsp90/Hsp70-based multiprotein chaperone machinery in reticulocyte lysate. J Biol Chem, 2001, 276: 30092-30098

23 Kosano H, Stensgard B, Charlesworth M C, et al. The assembly of progesterone receptor-Hsp90 complexes using purified proteins. J Biol Chem, 1998, 273: 32973-32979

24 Prodromou C. The ATPase cycle of Hsp90 drives a molecular 'clamp' via transient dimerization of the N-terminal domains. EMBO J, 2000, 19: 4383-4392

25 Vartholomaiou E, Echeverria P C, Picard D. Unusual suspects in the twilight zone between the Hsp90 interactome and carcinogenesis. Adv Cancer Res, 2016, 129: 1-30

26 Blagosklonny M V. Hsp-90-associated oncoproteins: Multiple targets of geldanamycin and its analogs. Leukemia, 2002, 16: 455-462

27 Taipale M, Jarosz D F, Lindquist S. HSP90 at the hub of protein homeostasis: Emerging mechanistic insights. Nat Rev Mol Cell Biol, 2010, 11: $515-528$

28 Giulino-Roth L, van Besien H J, Dalton T, et al. Inhibition of Hsp90 suppresses PI3K/AKT/mTOR signaling and has antitumor activity in Burkitt lymphoma. Mol Cancer Ther, 2017, 16: 1779-1790

29 Miao R Q, Fontana J, Fulton D, et al. Dominant-negative Hsp90 reduces VEGF-stimulated nitric oxide release and migration in endothelial cells. ATVB, 2008, 28: 105-111

30 Fortugno P, Beltrami E, Plescia J, et al. Regulation of survivin function by Hsp90. Proc Natl Acad Sci USA, 2003, 100: 13791-13796

31 Miyata Y, Nakamoto H, Neckers L. The therapeutic target Hsp90 and cancer hallmarks. Curr Pharm Des, 2013, 19: 347-365

32 Zhang R, Luo D, Miao R, et al. Hsp90-Akt phosphorylates ASK1 and inhibits ASK1-mediated apoptosis. Oncogene, 2005, 24: 3954-3963

33 Fulda S, Galluzzi L, Kroemer G. Targeting mitochondria for cancer therapy. Nat Rev Drug Discov, 2010, 9: 447-464

34 Whittier J E, Xiong Y, Rechsteiner M C, et al. Hsp90 enhances degradation of oxidized calmodulin by the 20S proteasome. J Biol Chem, 2004, 279: 46135-46142

35 Zhang Y, Kwon S H, Yamaguchi T, et al. Mice lacking histone deacetylase 6 have hyperacetylated tubulin but are viable and develop normally. MCB, 2008, 28: 1688-1701

36 Ai J, Wang Y, Dar J A, et al. HDAC6 regulates androgen receptor hypersensitivity and nuclear localization via modulating Hsp90 acetylation in castration-resistant prostate cancer. Mol Endocrinol, 2009, 23: 1963-1972

37 Zhang D, Li J, Costa M, et al. JNK1 mediates degradation HIF-1 by a VHL-independent mechanism that involves the chaperones Hsp90/Hsp70. Cancer Res, 2010, 70: 813-823

38 Mollapour M, Neckers L. Post-translational modifications of Hsp90 and their contributions to chaperone regulation. Biochim Biophys Acta, 2012, 1823: 648-655

39 Williams K A, Zhang M, Xiang S, et al. Extracellular signal-regulated kinase (ERK) phosphorylates histone deacetylase 6 (HDAC6) at serine 1035 to stimulate cell migration. J Biol Chem, 2013, 288: 33156-33170

40 Lin T Y, Fenger J, Murahari S, et al. AR-42, a novel HDAC inhibitor, exhibits biologic activity against malignant mast cell lines via downregulation of constitutively activated kit. Blood, 2010, 115: 4217-4225

41 Park J H, Kim S H, Choi M C, et al. Class II histone deacetylases play pivotal roles in heat shock protein 90-mediated proteasomal degradation of vascular endothelial growth factor receptors. Biochem Biophys Res Commun, 2008, 368: 318-322

42 Zhu J, Coyne C B, Sarkar S N. PKC alpha regulates Sendai virus-mediated interferon induction through HDAC6 and $\beta$-catenin. EMBO J, 2011, 30: 4838-4849

43 Lum M A, Balaburski G M, Murphy M E, et al. Heat shock proteins regulate activation-induced proteasomal degradation of the mature phosphorylated form of protein kinase C. J Biol Chem, 2013, 288: 27112-27127

44 Dallavalle S, Pisano C, Zunino F. Development and therapeutic impact of HDAC6-selective inhibitors. Biochem Pharmacol, 2012, 84: 756-765

45 Krämer O H, Stauber R H, Bug G, et al. SIAH proteins: Critical roles in leukemogenesis. Leukemia, 2013, 27: 792-802

46 Nishioka C, Ikezoe T, Yang J, et al. MS-275, a novel histone deacetylase inhibitor with selectivity against HDAC1, induces degradation of FLT3 via inhibition of chaperone function of heat shock protein 90 in AML cells. Leukemia Res, 2008, 32: 1382-1392

47 Li Y, Shin D, Kwon S H. Histone deacetylase 6 plays a role as a distinct regulator of diverse cellular processes. FEBS J, 2013, 280: 775-793

48 Yang Y, Rao R, Shen J, et al. Role of acetylation and extracellular location of heat shock protein 90 in tumor cell invasion. Cancer Res, 2008, 68: 4833-4842

49 Lafarga V, Aymerich I, Tapia O, et al. A novel GRK2/HDAC6 interaction modulates cell spreading and motility. EMBO J, 2012, 31: 856-869

50 Liu K P, Zhou D, Ouyang D Y, et al. LC3B-II deacetylation by histone deacetylase 6 is involved in serum-starvation-induced autophagic 
degradation. Biochem Biophys Res Commun, 2013, 441: 970-975

51 Boyault C, Gilquin B, Zhang Y, et al. HDAC6-p97/VCP controlled polyubiquitin chain turnover. EMBO J, 2006, 25: 3357-3366

52 Boyault C, Zhang Y, Fritah S, et al. HDAC6 controls major cell response pathways to cytotoxic accumulation of protein aggregates. Gene Dev, 2007, 21: 2172-2181

53 Kawaguchi Y, Kovacs J J, McLaurin A, et al. The deacetylase HDAC6 regulates aggresome formation and cell viability in response to misfolded protein stress. Cell, 2003, 115: 727-738

54 Fan Q, Yang L, Zhang X, et al. Autophagy promotes metastasis and glycolysis by upregulating MCT1 expression and Wnt/ $\beta$-catenin signaling pathway activation in hepatocellular carcinoma cells. J Exp Clin Cancer Res, 2018, 37: 9

55 Kaliszczak M, van Hechanova E, Li Y, et al. The HDAC6 inhibitor C1A modulates autophagy substrates in diverse cancer cells and induces cell death. Br J Cancer, 2018, 119: 1278-1287

56 Qiang L, Zhao B, Ming M, et al. Regulation of cell proliferation and migration by p62 through stabilization of Twist1. Proc Natl Acad Sci USA, 2014, 111: 9241-9246

57 Lopez G, Torres K, Lev D. Autophagy blockade enhances HDAC inhibitors' pro-apoptotic effects. Autophagy, 2011, 7: 440-441

58 Li J, Liu R, Lei Y, et al. Proteomic analysis revealed association of aberrant ROS signaling with suberoylanilide hydroxamic acid-induced autophagy in Jurkat T-leukemia cells. Autophagy, 2010, 6: 711-724

59 Thomas S, Thurn K T, Biçaku E, et al. Addition of a histone deacetylase inhibitor redirects tamoxifen-treated breast cancer cells into apoptosis, which is opposed by the induction of autophagy. Breast Cancer Res Treat, 2011, 130: 437-447

60 Jiang X, Huang Y, Liang X, et al. Metastatic prostate cancer-associated P62 inhibits autophagy flux and promotes epithelial to mesenchymal transition by sustaining the level of HDAC6. Prostate, 2018, 78: 426-434

61 Yan J, Seibenhener M L, Calderilla-Barbosa L, et al. SQSTM1/p62 interacts with HDAC6 and regulates deacetylase activity. PLoS One, 2013, 8: e76016-e76016

62 Shinojima N, Yokoyama T, Kondo Y, et al. Roles of the Akt/mTOR/p70S6K and ERK1/2 signaling pathways in curcumin-induced autophagy. Autophagy, 2007, 3: 635-637

63 Jiang P, Mizushima N. Autophagy and human diseases. Cell Res, 2014, 24: 69-79

64 Randhawa R, Sehgal M, Singh T R, et al. Unc-51 like kinase 1 (ULK1) in silico analysis for biomarker identification: A vital component of autophagy. Gene, 2015, 562: 40-49

65 Rout A K, Strub M P, Piszczek G, et al. Structure of transmembrane domain of lysosome-associated membrane protein type 2a (LAMP-2A) reveals key features for substrate specificity in chaperone-mediated autophagy. J Biol Chem, 2014, 289: 35111-35123

66 Xiao X, Wang W, Li Y, et al. HSP90AA1-mediated autophagy promotes drug resistance in osteosarcoma. J Exp Clin Cancer Res, 2018, 37: 201

67 Han J, Goldstein L A, Hou W, et al. HSP90 inhibition targets autophagy and induces a CASP9-dependent resistance mechanism in NSCLC. Autophagy, 2018, 14: 958-971

68 Aldana-Masangkay G I, Sakamoto K M. The role of HDAC6 in cancer. J Biomed Biotechnol, 2011, 2011: 875824

$69 \mathrm{Wu}$ J Y, Xiang S, Zhang M, et al. Histone deacetylase 6 (HDAC6) deacetylates extracellular signal-regulated kinase 1 (ERK1) and thereby stimulates ERK1 activity. J Biol Chem, 2018, 293: 1976-1993

70 Gao Y, Hubbert C C, Yao T P. The microtubule-associated histone deacetylase 6 (HDAC6) regulates epidermal growth factor receptor (EGFR) endocytic trafficking and degradation. J Biol Chem, 2010, 285: 11219-11226

$71 \mathrm{Gu} \mathrm{Y,} \mathrm{Xue} \mathrm{Q,} \mathrm{Chen} \mathrm{Y,} \mathrm{et} \mathrm{al.} \mathrm{Different} \mathrm{roles} \mathrm{of} \mathrm{PD-L1} \mathrm{and} \mathrm{FasL} \mathrm{in} \mathrm{immunomodulation} \mathrm{mediated} \mathrm{by} \mathrm{human} \mathrm{placenta-derived} \mathrm{mesenchymal} \mathrm{stem}$ cells. Human Immunol, 2013, 74: 267-276

72 Liu W, Fan L X, Zhou X, et al. HDAC6 regulates epidermal growth factor receptor (EGFR) endocytic trafficking and degradation in renal epithelial cells. PLoS One, 2012, 7: e49418-e49418

73 Wen J, Fu J, Ling Y, et al. MIIP accelerates epidermal growth factor receptor protein turnover and attenuates proliferation in non-small cell lung cancer. Oncotarget, 2016, 7: 9118-9134

74 Aoyagi S, Archer T K. Modulating molecular chaperone Hsp90 functions through reversible acetylation. Trends Cell Biol, 2005, 15: 565-567

75 Tsutsumi S, Beebe K, Neckers L. Impact of heat-shock protein 90 on cancer metastasis. Future Oncol, 2009, 5: 679-688

76 Choi Y J, Kim S Y, So K S, et al. AUY922 effectively overcomes MET- and AXL-mediated resistance to EGFR-TKI in lung cancer cells. PLoS One, 2015, 10: e0119832-e0119832

77 Tao H, Chen Y Y, Sun Z W, et al. Silence of HDAC6 suppressed esophageal squamous cell carcinoma proliferation and migration by disrupting chaperone function of HSP90. J Cell Biochem, 2018, 119: 6623-6632

78 Schulz R, Marchenko N D, Holembowski L, et al. Inhibiting the HSP90 chaperone destabilizes macrophage migration inhibitory factor and thereby inhibits breast tumor progression. J Exp Med, 2012, 209: 275-289

79 Yu S, Cai X, Wu C, et al. Targeting HSP90-HDAC6 regulating network implicates precision treatment of breast cancer. Int J Biol Sci, 2017, 13: 
505-517

80 Rosik L, Niegisch G, Fischer U, et al. Limited efficacy of specific HDAC6 inhibition in urothelial cancer cells. Cancer Biol Ther, 2014, 15: 742757

81 Rao R, Fiskus W, Yang Y, et al. HDAC6 inhibition enhances 17-AAG-mediated abrogation of Hsp90 chaperone function in human leukemia cells. Blood, 2008, 112: 1886-1893

82 Kim S H, Kang J G, Kim C S, et al. Novel heat shock protein 90 inhibitor NVP-AUY922 synergizes with the histone deacetylase inhibitor PXD101 in induction of death of anaplastic thyroid carcinoma cells. J Clin Endocrinol Metab, 2015, 100: E253-E261

83 Kim S H, Kang J G, Kim C S, et al. The heat shock protein 90 inhibitor SNX5422 has a synergistic activity with histone deacetylase inhibitors in induction of death of anaplastic thyroid carcinoma cells. Endocrine, 2016, 51: 274-282

84 Wang X X, Wan R Z, Liu Z P. Recent advances in the discovery of potent and selective HDAC6 inhibitors. Eur J Medicinal Chem, 2018, 143: $1406-1418$

85 Hoter A, El-Sabban M, Naim H. The HSP90 family: Structure, regulation, function, and implications in health and disease. Int J Mol Sci, 2018, 19: 2560

86 George P, Bali P, Annavarapu S, et al. Combination of the histone deacetylase inhibitor LBH589 and the Hsp90 inhibitor 17-AAG is highly active against human CML-BC cells and AML cells with activating mutation of FLT-3. Blood, 2005, 105: 1768-1776

87 Bali P, Pranpat M, Bradner J, et al. Inhibition of histone deacetylase 6 acetylates and disrupts the chaperone function of heat shock protein 90 . J Biol Chem, 2005, 280: 26729-26734

88 Nimmanapalli R, Fuino L, Bali P, et al. Histone deacetylase inhibitor LAQ824 both lowers expression and promotes proteasomal degradation of $\mathrm{Bcr}-\mathrm{Abl}$ and induces apoptosis of imatinib mesylate-sensitive or -refractory chronic myelogenous leukemia-blast crisis cells. Cancer Res, 2003, 63: 5126-5135

89 Bali P. Superior activity of the combination of histone deacetylase inhibitor LAQ824 and the FLT-3 kinase inhibitor PKC412 against human acute myelogenous leukemia cells with mutant FLT-3. Clin Cancer Res, 2004, 10: 4991-4997

90 Neckers L, Blagg B, Haystead T, et al. Methods to validate Hsp90 inhibitor specificity, to identify off-target effects, and to rethink approaches for further clinical development. Cell Stress Chaperones, 2018, 23: 467-482 


\title{
HDAC6-HSP90 pathway-mediated protein folding and degra- dation regulates tumor cell proliferation and metastasis
}

\author{
Rui Tian ${ }^{\dagger}$, Xiaoyu Yan ${ }^{\dagger}$, Sihang Yu, Long Xu, Yuanxin Zhao, Liankun Sun ${ }^{*} \&$ Jing Su* \\ Key Laboratory of Pathophysiology, Ministry of Education, Department of Pathophysiology, Basic Medical College, Jilin University, Changchun \\ 130000, China \\ $\dagger$ Equally contributed to this work \\ * Corresponding authors, E-mail: sunlk@jlu.edu.cn; sujing@jlu.edu.cn
}

The folding and degradation of proteins in tumor cells are the key mechanisms maintaining their survival, and functions of the proteins involved are often related to the prevailing tumor resistance. With further scientific research, an increasing number of auxiliary proteins involved in protein folding and degradation have been reported in the literature. The chaperone heat shock protein 90 (HSP90) not only promotes protein folding and plays a role in maintaining protein conformation stability but also assists the proteasomal degradation of client proteins. Current research suggests that the function of HSP90 is regulated by post-translational modifications, such as acetylation. Histone deacetylase 6 (HDAC6) can inhibit the over-acetylation of HSP90 and maintain the chaperone function of HSP90 to ensure that the client protein of the chaperone HSP90 can be stably folded and maturely released, which creates conditions for tumor survival, proliferation, and metastasis. Furthermore, it can act as the client protein and autophagy receptor of HSP90. It recruits ubiquitinated proteins and deacetylates and modifies autophagy-related proteins, thus playing important roles in protein degradation and autophagy. Recently, studies revealed HDAC6, a client protein of HSP90, which plays an important role in protein degradation, cell autophagy, and maintenance of the activity of HSP90 by preventing its excessive acetylation. By inhibiting HDAC6 to promote HSP90 over-acetylation, it can effectively inhibit the binding between HSP90 and ATP, thereby affecting the folding and degradation of HSP90 on tumor proliferation and metastasis-related proteins. Simultaneously, HDAC inhibitors can help HSP90 bind more closely to its inhibitors. Therefore, HDAC6 and HSP90 inhibitors have also been widely used in related research on tumor treatment, and studies have shown that the combined application of HDAC6 and HSP90 inhibitors has a significant effect on inhibiting tumor proliferation and metastasis. Whether it is to inhibit HDAC6 alone or HSP90 alone, there are deficiencies in cancer treatment. The use of inhibitors alone may lead to the activation of other pathways related to tumor cell proliferation and survival. HDAC6-HSP90 dualtarget inhibition can reduce the activation of compensatory pathways, such as autophagy, and reduce the possibility of offtarget effects of HSP90 inhibitors. In this review, we focus on the crosstalk between HDAC6 and HSP90 to further explain how tumor cells initiate proliferation and metastasis signals by regulating protein folding and degradation mechanisms and discuss the research applications of HDAC6 and HSP90 inhibitors. The advantages and disadvantages are assessed to obtain novel strategies for cancer therapy by targeting the HSP90-HDAC6 pathway.

HDAC6, HSP90, protein folding and degradation, tumor proliferation and metastasis doi: 10.1360/TB-2020-0644 